\title{
Phenethyl Isothiocyanate-containing Watercress Juice
}

National Cancer Institute

\section{Source}

National Cancer Institute. Phenethyl Isothiocyanate-containing Watercress /uice. NCI

Thesaurus. Code C105392.

A juice extracted from watercress containing high amounts of phenethyl isothiocyanate (PEITC), with potential chemopreventive and antitumor activities. Although the mechanism(s) of action through which PEITC exerts its effect(s) has yet to be fully elucidated, PEITC is able to induce apoptosis in tumor cells through the induction of reactive oxygen species (ROS). Additionally, PEITC is able to modulate extracellular signalregulated kinases (ERK), c-Jun N-terminal kinase (JNK) and mitogen-activated protein kinase (MAPK) signal transduction pathways, activating the expression of stressresponsive genes and eventually inducing apoptosis. PEITC also inhibits the expression of genes involved in tumor progression such as HIF, STAT-3, HER2, BCL-XL, and XIAP and induces the expression of genes involved in tumor suppression such as p53, ATF-2, and p57. Furthermore, this agent has been shown to reactivate the gene expression of certain detoxification enzymes. 